\title{
Qualidade do palmito da palmeira real em conserva
}

\author{
Industrial adaptability of the heart of palm from Archontophoenix alexandrae and A. cunninghamiana
}

\author{
Shirley Aparecida Garcia BERBARI ${ }^{1 *}$, Patricia PRATI ${ }^{1}$, Valéria Christina Amstalden JUNQUEIRA ${ }^{1}$
}

\begin{abstract}
Resumo
A pesquisa teve como objetivo avaliar o aproveitamento industrial e a qualidade do palmito da palmeira Real Australiana (Archontophoenix alexandrae e A. cunninghamiana) em comparação aos palmitos das palmeiras Açaí (Euterpe oleracea) e Pupunha (Bactris gasipaes). O palmito foi processado como conserva acidificada e pasteurizada e submetido às análises de comprimento e diâmetro dos toletes, peso bruto, líquido e drenado, espaço livre, vácuo, $\mathrm{pH}$, avaliação microbiológica, cor e textura objetivas e avaliação sensorial quanto à cor, aparência, textura, sabor e preferência geral. Os resultados da pesquisa mostraram que o palmito da palmeira Real é adequado para processamento em forma de conserva acidificada e pasteurizada, apresentando características sensoriais semelhantes às das outras variedades. Do ponto de vista da estabilidade ao armazenamento, as análises físico-químicas e microbiológicas mostraram que o palmito da palmeira Real em conserva se apresentou estável durante o período de doze meses de armazenamento. Pode-se afirmar então, que a palmeira Real Australiana apresenta boa qualidade em termos de industrialização na forma de palmito em conserva acidificada e pasteurizada.

Palavras-chave: palmito; acidificação; pasteurização; estabilidade; armazenamento.
\end{abstract}

\begin{abstract}
The objective of this research was to evaluate the industrial adaptability of the heart of palm from Archontophoenix alexandrae e A. cunninghamiana in comparison with "Açaî" (Euterpe oleracea) and "Pupunha" (Bactris gasipaes) hearts. The heart of palm was canned acidified and pasteurized and analyzed in terms of stems length and diameter, the gross, net and drained weight, headspace; pH; vacuum; microbiological evaluation, objective color and texture and sensorial evaluation of color, appearance, texture, taste and general preference. The results showed that Australian royal palm is adequate for the process of acidified canning and pasteurization since it presented sensorial characteristics similar to other varieties. During 12 months of storage, the physicochemical analysis showed that the Australian royal palm remained stable. It can be said that the Australian royal palm is adequate for industrialization as a canned acidified and pasteurized product.

Keywords: heart of palm; acidification; pasteurization; stability; storage.
\end{abstract}

\section{Introdução}

O Brasil destaca-se quanto à industrialização de palmito em conserva acidificada e pasteurizada tanto pela quantidade processada quanto por suas pesquisas nesta área.

Antes da década de 60 a produção básica de palmito vinha principalmente da costa meridional do país, sendo extraído da palmeira Juçara. O Estado de São Paulo era então o primeiro produtor. O ritmo da exploração, sem o correspondente replantio, fez cair rapidamente o número de palmeiras nativas nessa região. Esta escassez de matéria-prima acarretou a mudança das maiores empresas processadoras de palmito para o estado do Pará, então com extensas reservas de açaizeiros (BOVI, 1997).

O uso racional de outras palmeiras para a produção de palmito tem sido uma das alternativas para diminuir a pressão de exploração sobre as espécies Euterpe edulis e Euterpe oleracea (BOVI, 1998). Dentre as várias palmeiras passíveis de serem cultivadas para esta finalidade, merece destaque a palmeira Real Australiana, Archontophoenix alexandrae e A. cunninghamiana, devido principalmente às suas características de precocidade $\mathrm{e}$ rusticidade. Esta palmeira era utilizada em nosso país até recentemente, apenas como planta ornamental. $\mathrm{O}$ interesse por esta palmeira como produtora de palmito começou a partir dos anos 90, quando a exploração predatória da palmeira Juçara na região Sudeste do Brasil e do Açaí no Norte tinham alcançado o máximo e nossas reservas de palmito nativo já estavam bastante dilapidadas. Estudos efetuados com plantas dessas espécies atestam a viabilidade de seu cultivo em nosso meio, ou seja, no Brasil (BOVI, 1998).

A produção de palmito nas espécies Archontophoenix é feita a partir de dois anos de campo, desde que cultivadas em regiões aptas e com adubação apropriada. As espécies tradicionais levam de 8 a 12 anos para estarem prontas para o corte. Por ser recomendada para plantio em pleno sol, elimina os problemas que os proprietários rurais têm enfrentado com a legislação existente para áreas cobertas com matas naturais, possibilitando ainda maior tecnificação da cultura, através da mecanização (BOVI, 1998).

A qualidade do palmito em conserva foi estudada por Mori et al. (1989). Foram identificados e quantificados diversos atributos de qualidade: cor, uniformidade de diâmetro e comprimento, defeitos, aspecto da salmoura, aparência geral,

${ }^{1}$ Instituto de Tecnologia de Alimentos - ITAL, Av. Brasil, 2880, Chapadão, CEP 13070-180, Campinas - SP, Brasil, E-mail: sberbari@ital.sp.gov.br

${ }^{*}$ A quem a correspondência deve ser enviada 
odor, maciez, fibrosidade, gosto ácido, sabor de palmito, sabor estranho e grau de qualidade.

Ferreira et al. (1981; 1982) avaliaram o palmito de Pupunha em comparação com o palmito de Juçara, considerado de melhor qualidade e maior valor comercial. Ao contrário do palmito de Juçara, o palmito de Pupunha não apresentou atividade enzimática, tendo apresentado, após o processamento, cor amarelada e boa aceitação.

O presente trabalho de pesquisa teve como objetivo avaliar o aproveitamento industrial e a qualidade do palmito da palmeira Real Australiana (Archontophoenix alexandrae e A. cunninghamiana) em comparação aos palmitos das palmeiras Açaí (Euterpe oleracea) e Pupunha (Bactris gasipaes).

\section{Material e métodos}

\subsection{Matéria-prima}

Foram utilizadas palmeiras Açaí (Euterpe oleracea), Pupunha (Bactris gasipaes) e Real Australiana (Archontophoenix alexandrae e A. cunninghamiana) provenientes da estação experimental do IAC, localizada em Ubatuba - SP, região do litoral norte de SP e colhidas com quatro anos de plantio.

\section{Curva de acidificação}

Para realização da curva de acidificação foram retirados seis talos de palmito do lote a ser processado. A análise foi realizada conforme descrito por Zapata e Quast (1975). As curvas de acidificação foram calculadas para cada amostra, expressando o poder tamponante em gramas de ácido cítrico para $100 \mathrm{~g}$ de palmito fresco quando em $\mathrm{pH} 4,3$, inferior a 4,5, considerado o limite máximo de segurança pela Legislação Brasileira conforme a Resolução - RDC No 17 de 19/11/99 - ANVISA/MS.

\section{Caracterização físico-química}

Nas amostras de palmito in natura das espécies avaliadas foram realizadas análises de:

- Teor de umidade, segundo metodologia descrita pelo Instituto Adolfo Lutz (2005);

- Cinzas, segundo metodologia descrita em AOAC (2000);

- Lipídios Totais, segundo metodologia descrita em AOAC (2000);

- Açúcares Totais e Redutores, segundo metodologia descrita em AOAC (2000);

- Proteínas, segundo metodologia descrita em AOAC (1997);

- Acidez Total Titulável, segundo metodologia descrita em AOAC (2000); e

- Amido, segundo metodologia descrita por Diemair (1963).

\subsection{Metodologia}

\section{Processamento}

O processamento dos palmitos em forma de conserva acidificada e pasteurizada foi realizado conforme descrito no fluxograma mostrado pela Figura 1.

O desembainhamento parcial para a retirada das capas externas foi executado com facões de aço e o desembainhamento final com facas de aço inoxidável, sendo retiradas duas ou três bainhas internas restantes. $\mathrm{O}$ corte foi realizado com facas de aço inoxidável e com o auxílio de um gabarito para padronização do comprimento dos toletes em $9 \mathrm{~cm}$. O critério para o corte dos toletes foi baseado na resistência oferecida pela matéria-prima ao corte, ou seja, enquanto a textura do tolete ofereceu suave resistência à penetração da faca, este foi considerado comestível.

Após o corte, os toletes foram imersos em solução de espera, preparada tal qual a salmoura de enchimento utilizada no acondicionamento dos toletes. $\mathrm{O}$ acondicionamento foi realizado em vidros com capacidade para $600 \mathrm{~mL}$, onde foram acondicionados $320 \mathrm{~g}$ de palmito e $250 \mathrm{~g}$ de salmoura acidificada com ácido cítrico em concentração calculada de acordo com

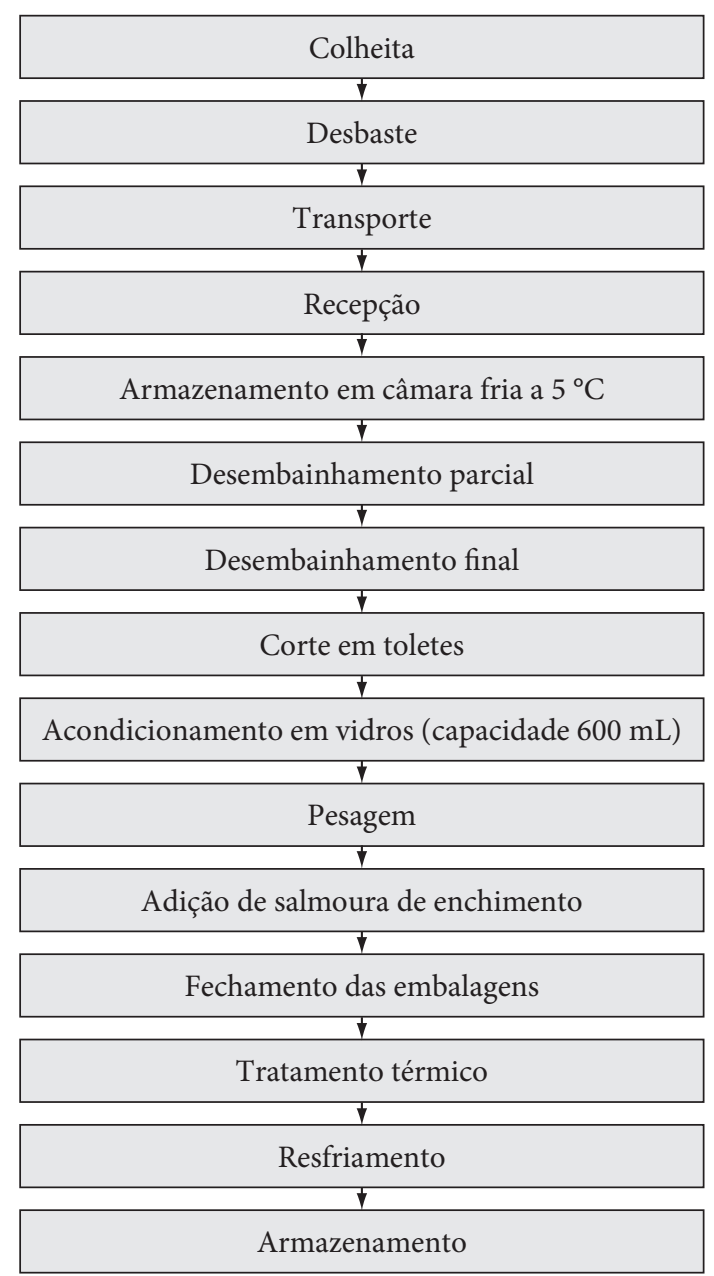

Figura 1. Etapas básicas do processamento do palmito em conserva. 
os resultados obtidos na curvas de acidificação da matériaprima, obtidas conforme descrito por Zapata e Quast (1975). $\mathrm{O}$ tratamento térmico para pasteurização do produto foi feito em tanques com água em ebulição durante 45 minutos, seguido do resfriamento em água corrente até o centro das embalagens atingirem aproximadamente $37^{\circ} \mathrm{C}$. O armazenamento do produto foi realizado em lugar seco, ventilado, e à temperatura ambiente $\left(25^{\circ} \mathrm{C}\right)$.

\section{Análises físicas e físico-químicas}

Os palmitos em conserva foram analisados em sete épocas durante o período de 12 meses de armazenamento, de acordo com as seguintes determinações:

- Comprimento e diâmetro dos toletes: em paquímetro, com resultados expressos em milímetros;

- Determinação de peso: em balança semi-analítica, com resultados expressos em gramas;

- Espaço livre: em régua específica, com resultados expressos em milímetros;

- $\mathrm{pH}$ de equilíbrio: em potenciômetro marca Digimed DM-20 (AOAC, 1997); e

- Vácuo: em vacuômetro, com resultados expressos em pol de Hg.

\section{Avaliação microbiológica}

Os palmitos em conserva foram submetidos à avaliação de esterilidade comercial, 45 dias após o processamento, segundo metodologia descrita por Downes (2001).

\section{Avaliação objetiva da cor}

Foi realizada em colorímetro marca Minolta Chroma Meter CR - 300, sistema Hunter Lab, com resultados expressos em valor $\mathrm{L}($ luminosidade), $\mathrm{a}($ negativo $=$ verde, positivo $=$ vermelho $) \mathrm{e}$ $\mathrm{b}($ positivo $=$ amarelo, negativo $=$ azul). As leituras em triplicata foram realizadas em sete épocas de avaliação durante 12 meses de armazenamento do produto. Os resultados foram analisados estatisticamente através da análise de variância (teste F) e Teste de Tukey (diferença entre as médias) a 5\% de significância. Foram avaliados os toletes inteiros, para avaliação da cor externa, e cortados transversalmente, para avaliação da cor interna.

\section{Avaliação de textura}

Foi realizada por meio da determinação de resistência ao corte em texturômetro TAX-T2i, marca SMS, operando com software Texture Expert, com modo e operação de força de compressão, opção return to start, velocidade de pré-teste $5 \mathrm{~mm} / \mathrm{s}$, velocidade de teste $1 \mathrm{~mm} / \mathrm{s}$ e velocidade de pós-teste $5 \mathrm{~mm} / \mathrm{s}$. Utilizou-se um corpo de prova (probe), blade set with $k n i f e$, com curso de penetração de $40 \mathrm{~mm}$. As amostras foram dispostas na célula de teste de duas maneiras diferentes. $\mathrm{Na}$ primeira, os toletes foram colocados inteiros na célula e a faca descia transversalmente à fibra. Na segunda, os toletes foram cortados em rodelas, colocadas na célula de forma que a lâmina descia longitudinalmente à fibra. As épocas de avaliação encontram-se citadas nos itens (Análises físicas e físico-químicas) e (Avaliação objetiva da cor). Os resultados foram expressos em gf (grama força).

\section{Avaliação sensorial}

Os palmitos em conserva foram avaliados subjetivamente quanto à cor, aparência, textura, sabor e preferência geral. Foi utilizada uma equipe de 12 provadores treinados e uma escala hedônica estruturada com sete pontos, variando entre os termos "gostei muitíssimo" (7) e "desgostei muitíssimo" (1) (ISO, 1983). Por se tratar de produto em conserva, com estimativa de vida de prateleira longa, as análises foram realizadas em quatro épocas de avaliação, durante o período de um ano de armazenamento do produto. Utilizou-se o delineamento experimental de blocos completos casualizados. Os resultados foram analisados através da Análise de Variância (teste $F$ ) e Teste de Tukey (diferença entre as médias) a 5\% de significância (FARIA; YOTSUYANAGI, 2002).

\section{Resultados e discussão}

\subsection{Curvas de acidificação}

As curvas de acidificação dos palmitos das diferentes palmeiras estão ilustradas na Figura 2.

De acordo com a Figura 2, para acidificar até pH 4,3, limite segurança estabelecido no processamento industrial, $100 \mathrm{~g}$ de palmito das espécies Açaí e Pupunha, foram necessários 0,412 g

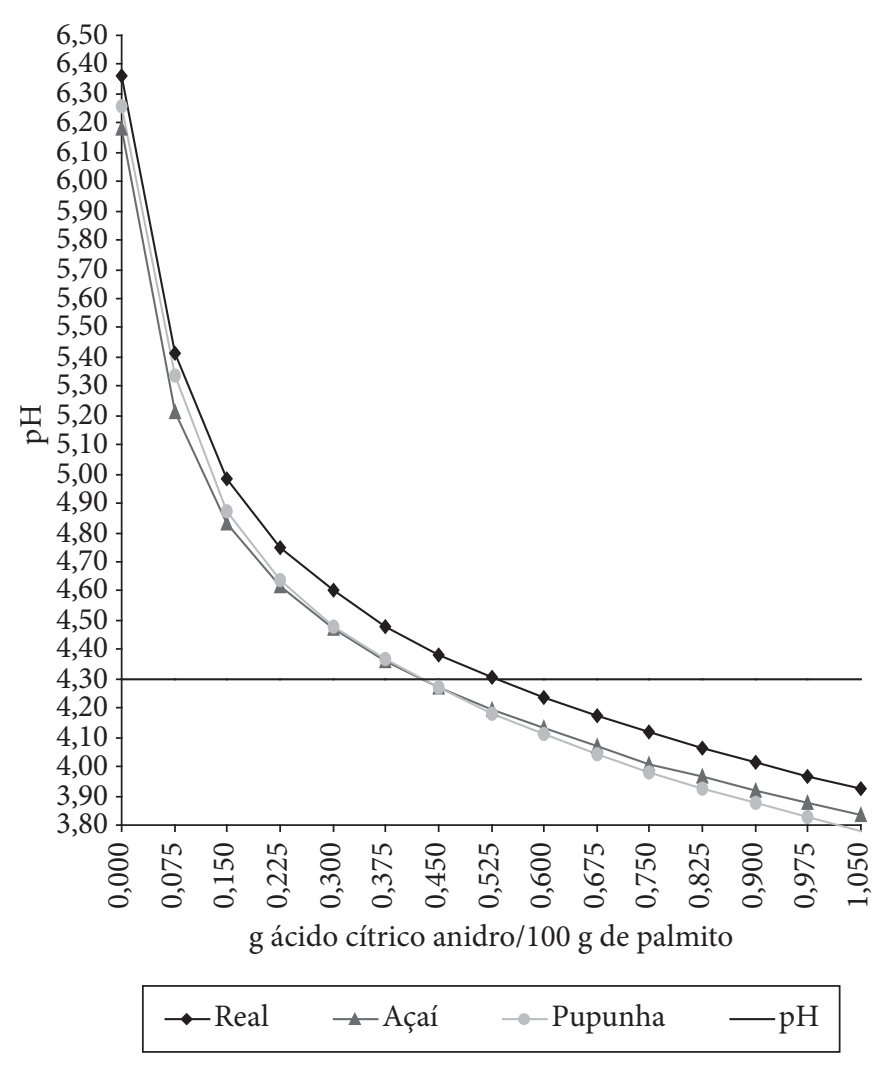

Figura 2. Curvas de acidificação de palmitos de diferentes palmeiras. 
de ácido cítrico, enquanto que para o palmito da palmeira Real foram necessários $0,525 \mathrm{~g}$ de ácido cítrico. Esta variação de $27 \%$ na quantidade de ácido necessária para acidificar o palmito das diferentes espécies de palmeiras tem importância do ponto de vista de segurança alimentar do produto, pois a correta acidificação para $\mathrm{pH}$ menor ou igual a 4,5 é a garantia de que no palmito em conserva não irão se desenvolver os esporos do Clostridium botulinum.

\subsection{Análises físico-químicas}

Os resultados da caracterização físico-química do palmito in natura estão apresentados na Tabela 1.

Tabela 1. Resultados da caracterização físico-química do palmito in natura.

\begin{tabular}{|c|c|c|c|}
\hline Parâmetros & Real & Açaí & Pupunha \\
\hline Umidade (g.100 g $\left.\mathrm{g}^{-1}\right)$ & $91,5 \pm 0,0$ & $92,5 \pm 0,0$ & $92,2 \pm 0,0$ \\
\hline Cinzas $\left(\mathrm{g} .100 \mathrm{~g}^{-1}\right)$ & $0,9 \pm 0,0$ & $1,1 \pm 0,0$ & $0,8 \pm 0,0$ \\
\hline Lipídios totais $\left(\mathrm{g} .100 \mathrm{~g}^{-1}\right)$ & $0,5 \pm 0,0$ & $0,5 \pm 0,0$ & $0,5 \pm 0,0$ \\
\hline Açúcares totais $\left(\mathrm{g} .100 \mathrm{~g}^{-1}\right)$ & $1,6 \pm 0,0$ & $0,2 \pm 0,0$ & $0,9 \pm 0,0$ \\
\hline Açúcares redutores $\left(\mathrm{g} .100 \mathrm{~g}^{-1}\right)$ & $1,2 \pm 0,0$ & $0,1 \pm 0,0$ & $0,7 \pm 0,1$ \\
\hline Proteína $\left(\mathrm{N} x\right.$ 5,75) $\left(\mathrm{g} .100 \mathrm{~g}^{-1}\right)$ & $2,5 \pm 0,0$ & $2,5 \pm 0,0$ & $2,5 \pm 0,0$ \\
\hline Acidez total (mL NaOH $\left.1 \mathrm{~N} .100 \mathrm{~g}^{-1}\right)$ & $0,9 \pm 0,0$ & $0,5 \pm 0,0$ & $1,1 \pm 0,0$ \\
\hline Carboidratos $^{\mathrm{a}}\left(\mathrm{g} .100 \mathrm{~g}^{-1}\right)$ & 4,6 & 3,4 & 4,0 \\
\hline Calorias $^{\mathrm{b}}\left(\mathrm{kcal} 100 \mathrm{~g}^{-1}\right)$ & 33 & 28 & 31 \\
\hline Amido (g.100 g-1) & $1,0 \pm 0,0$ & $0,5 \pm 0,1$ & $1,0 \pm 0,0$ \\
\hline
\end{tabular}

${ }^{a}$ Calculado por diferença: $100-\left(\mathrm{g} .100 \mathrm{~g} \mathrm{~g}^{-1}\right.$ umidade $+\mathrm{g} .100 \mathrm{~g}^{-1}$ cinzas + g. $100 \mathrm{~g}^{-1}$ proteína bruta + g. $100 \mathrm{~g} \mathrm{~g}^{-1}$ lipídios totais); $\mathrm{e}^{\mathrm{b}} \mathrm{O}$ valor calórico da amostra foi calculado pela soma das porcentagens de proteína bruta e carboidratos multiplicados pelo fator $4\left(\mathrm{kcal}^{-\mathrm{g}^{-1}}\right)$ somado ao teor de lipídios totais multiplicado pelo fator $9\left(\mathrm{kcal} . \mathrm{g}^{-1}\right)$.
Os resultados apresentados na Tabela 1, quando comparados com aqueles obtidos por Ferreira et al. (1981; 1982), indicam que os palmitos de Pupunha e Juçara apresentaram teores levemente maiores de umidade, matéria graxa e cinzas; e teores de proteína semelhantes. Observando-se os resultados obtidos para as três variedades estudadas, verifica-se grande variação no teor de açúcares totais, sendo que o palmito Real apresentou quantidades de açúcares totais 1,8 e 8 vezes maiores que os palmitos Pupunha e Açaí, respectivamente.

\subsection{Análises físicas e físico-químicas}

Os resultados das análises dos parâmetros de qualidade do palmito em conserva, em diferentes épocas, estão apresentados na Tabela 2. De acordo com esses resultados, todas as amostras analisadas encontram-se dentro dos parâmetros de segurança alimentar exigidos pela Legislação Brasileira, conforme a Resolução - RDC No 17 de 19/11/99 - ANVISA/ MS, apresentando valores de vácuo superiores a 10 pol. de $\mathrm{Hg}$ e pH de equilíbrio inferior a 4,5. Vale ressaltar que durante o período de armazenamento avaliado não foram verificadas alterações nestes parâmetros, indicando estabilidade do palmito em conserva por um período de 360 dias (1 ano) quanto às características físico-químicas.

\subsection{Avaliação microbiológica}

Os resultados do teste de esterilidade comercial do palmito em conserva revelaram ausência de microrganismos mesófilos e termófilos acidotolerantes, bolores e leveduras, ou seja, as amostras encontraram-se de acordo com os padrões microbiológicos estabelecidos pela Resolução RDC Número 12 de 01 de janeiro

Tabela 2. Parâmetros de qualidade das conservas de palmito em diferentes épocas de armazenamento.

\begin{tabular}{|c|c|c|c|c|c|c|c|c|c|c|c|c|}
\hline \multirow[t]{2}{*}{ Parâmetros } & \multicolumn{3}{|c|}{$1^{\text {a época }}$} & \multicolumn{3}{|c|}{$2^{\mathrm{a}}$ época } & \multicolumn{3}{|c|}{$3^{a}$ época } & \multicolumn{3}{|c|}{$4^{\mathrm{a}}$ época } \\
\hline & Real & Açaí & Pupunha & Real & Açaí & Pupunha & Real & Açaí & Pupunha & Real & Açaí & Pupunha \\
\hline Comprimento $(\mathrm{mm})$ & 87,0 & 86,0 & 88,0 & 87,0 & 85,0 & 87,0 & 87,0 & 86,0 & 86,0 & 87,0 & 86,0 & 87,0 \\
\hline Diâmetro (mm) & 32,0 & 35,0 & 28,0 & 31,0 & 35,0 & 27,0 & 28,0 & 37,0 & 30,0 & 37,0 & 36,0 & 28,0 \\
\hline Peso bruto (g) & 875,0 & 872,8 & 875,3 & 871,3 & 879,0 & 874,0 & 869,2 & 865,2 & 878,8 & 872,0 & 868,9 & 876,5 \\
\hline Peso drenado (g) & 322,4 & 310,3 & 305,4 & 323,6 & 303,1 & 320,7 & 322,6 & 311,5 & 311,0 & 319,2 & 317,1 & 314,0 \\
\hline Peso da embalagem (g) & 325,7 & 327,1 & 325,2 & 325,9 & 327,0 & 326,1 & 325,3 & 328,0 & 326,5 & 324,8 & 326,1 & 326,3 \\
\hline Peso líquido (g) & 549,3 & 550,2 & 550,1 & 545,5 & 552,1 & 547,9 & 543,9 & 537,2 & 552,3 & 547,2 & 542,8 & 550,2 \\
\hline Peso da salmoura (g) & 226,9 & 239,9 & 244,8 & 221,9 & 249,1 & 227,2 & 221,3 & 225,8 & 241,3 & 228,1 & 225,6 & 236,2 \\
\hline Espaço livre (mm) & 12,5 & 13,5 & 13,0 & 15,0 & 12,0 & 14,0 & 15,5 & 17,0 & 14,0 & 14,5 & 16,0 & 14,0 \\
\hline pH de equilíbrio & 4,1 & 4,2 & 4,1 & 4,1 & 4,2 & 4,2 & 4,1 & 4,1 & 4,1 & 4,0 & 4,2 & 4,2 \\
\hline \multirow[t]{3}{*}{ Vácuo (pol Hg) } & 18,8 & 19,8 & 20,5 & 18,3 & 17,3 & 16,8 & 19,5 & 19,5 & 18,8 & 17,3 & 19,8 & 18,0 \\
\hline & \multicolumn{3}{|c|}{$5^{a}$ época } & \multicolumn{3}{|c|}{$6^{a}$ época } & \multicolumn{3}{|c|}{$7^{\mathrm{a}}$ época } & & & \\
\hline & Real & Açaí & Pupunha & Real & Açaí & Pupunha & Real & Açaí & Pupunha & & & \\
\hline Comprimento (mm) & 85,0 & 86,0 & 85,0 & 93,0 & 91,0 & 86,0 & 87,0 & 85,0 & 86,0 & & & \\
\hline Diâmetro (mm) & 33,0 & 34,0 & 28,0 & 36,0 & 41,0 & 26,0 & 31,0 & 35,0 & 27,0 & & & \\
\hline Peso bruto (g) & 878,2 & 855,5 & 882,4 & 869,6 & 871,0 & 869,6 & 882,3 & 873,1 & 873,1 & & & \\
\hline Peso drenado (g) & 323,8 & 316,8 & 311,3 & 322,0 & 313,9 & 309,5 & 317,1 & 315,8 & 315,2 & & & \\
\hline Peso da embalagem (g) & 325,1 & 327,1 & 326,3 & 325,7 & 327,6 & 325,1 & 325,6 & 327,6 & 326,4 & & & \\
\hline Peso líquido (g) & 553,1 & 528,4 & 556,1 & 543,9 & 543,4 & 544,5 & 556,7 & 545,5 & 546,7 & & & \\
\hline Peso da salmoura (g) & 229,3 & 211,6 & 244,8 & 222,0 & 229,5 & 235,0 & 239,5 & 229,8 & 231,4 & & & \\
\hline Espaço livre (mm) & 13,0 & 19,0 & 12,5 & 16,0 & 15,0 & 15,0 & 12,5 & 15,0 & 15,0 & & & \\
\hline pH de equilíbrio & 4,0 & 4,2 & 4,1 & 4,0 & 4,1 & 4,1 & 3,9 & 4,2 & 4,2 & & & \\
\hline Vácuo (pol Hg) & 16,3 & 19,0 & 18,5 & 19,3 & 19,8 & 19,0 & 13,8 & 18,5 & 18,0 & & & \\
\hline
\end{tabular}


de 2001, da Agência Nacional de Vigilância Sanitária (ANVISA), sendo consideradas comercialmente estéreis.

\subsection{Avaliação objetiva da cor}

Os resultados da análise objetiva de cor nas diferentes épocas estão apresentados nas Tabelas 3, 4 e 5.

Os valores do parâmetro L (luminosidade) mostram que, quanto à superfície do tolete e da rodela, todas as espécies estudadas nas diferentes épocas de avaliação, embora semelhantes, diferiram significativamente entre si durante o período de armazenamento. Segundo Tauszig (2000), quanto maior o valor de L, mais clara a cor do produto. A diminuição nos valores de L indica que o palmito em conserva apresentou leve escurecimento durante o período de armazenamento. Quanto ao parâmetro "a" (negativo $=$ verde, positivo $=$ vermelho $)$, todos os valores encontrados foram negativos, indicando traços de cor verde nos palmitos em conserva das três espécies estudadas.

Durante o armazenamento, houve perda de coloração verde em todas as variedades estudadas, indicando a provável oxidação dos pigmentos e conseqüente escurecimento das mesmas. Para o parâmetro "a" também foi verificada diferença significativa entre as variedades durante o período de armazenamento. Para o parâmetro "b" (positivo = amarelo, negativo = azul) foram encontrados valores positivos indicando coloração amarela para todas as amostras avaliadas. Assim sendo, a coloração do palmito em conserva resulta da composição da cor verde e amarela. Os resultados obtidos para cor objetiva (L, a e b) dos palmitos em conserva são concordantes e indicam leve escurecimento do produto durante os 12 meses de avaliação. Para o palmito Pupunha, os teores de verde (a negativo) e amarelo (b positivo) mais elevados indicam coloração amarelada para esta espécie. Já

Tabela 3. Parâmetro L referente à cor das conservas de palmito (médias de três repetições analíticas).

\begin{tabular}{|c|c|c|c|c|c|c|c|}
\hline \multirow[t]{2}{*}{ Variedade } & \multicolumn{7}{|c|}{ Cor externa } \\
\hline & $1^{\mathrm{a}}$ época & $2^{\mathrm{a}}$ época & $3^{\mathrm{a}}$ época & $4^{\mathrm{a}}$ época & $5^{\mathrm{a}}$ época & $6^{\mathrm{a}}$ época & $7^{\mathrm{a}}$ época \\
\hline Real & $83,13^{\mathrm{a}, \mathrm{B}}$ & $79,91^{\mathrm{abc}, \mathrm{B}}$ & $81,29^{\mathrm{ab}, \mathrm{B}}$ & $79,43^{\mathrm{bc}, \mathrm{B}}$ & $78,02^{\mathrm{c}, \mathrm{B}}$ & $79,03^{\mathrm{bc}, \mathrm{B}}$ & $78,68^{\mathrm{bc}, \mathrm{I}}$ \\
\hline Açaí & $85,71^{\mathrm{a}, \mathrm{A}}$ & $84,84^{\mathrm{ab}, \mathrm{A}}$ & $85,00^{\mathrm{ab}, \mathrm{A}}$ & $84,86^{\mathrm{ab}, \mathrm{A}}$ & $85,22^{\mathrm{ab}, \mathrm{A}}$ & $84,45^{\mathrm{ab}, \mathrm{A}}$ & $83,15^{\mathrm{b}, \mathrm{A}}$ \\
\hline \multirow[t]{2}{*}{ Pupunha } & $80,33^{\mathrm{a}, \mathrm{C}}$ & $79,68^{\mathrm{ab}, \mathrm{B}}$ & $77,57^{\mathrm{bc}, \mathrm{C}}$ & $76,95^{\mathrm{c}, \mathrm{C}}$ & $77,84^{\mathrm{abc}, \mathrm{B}}$ & $77,46^{\mathrm{bc}, \mathrm{B}}$ & $77,27^{\mathrm{bc} \mathrm{B}}$ \\
\hline & \multicolumn{7}{|c|}{ Cor interna } \\
\hline Real & $79,91^{\mathrm{a}, \mathrm{B}}$ & $78,13^{\mathrm{ab}, \mathrm{A}}$ & $76,55^{\mathrm{ab}, \mathrm{B}}$ & $76,21^{\mathrm{ab}, \mathrm{B}}$ & $76,69^{\mathrm{ab}, \mathrm{B}}$ & $78,69^{\mathrm{ab}, \mathrm{A}}$ & $75,41^{\mathrm{b}, \mathrm{B}}$ \\
\hline Açaí & $83,98^{\mathrm{a}, \mathrm{A}}$ & $81,68^{\mathrm{ab}, \mathrm{A}}$ & $81,24^{\mathrm{ab}, \mathrm{A}}$ & $80,20^{\mathrm{b}, \mathrm{A}}$ & $80,63^{\mathrm{ab}, \mathrm{A}}$ & $80,52^{\mathrm{ab}, \mathrm{A}}$ & $78,67^{\mathrm{b}, \mathrm{A}}$ \\
\hline Pupunha & $82,16^{\mathrm{a}, \mathrm{AB}}$ & $79,95^{\mathrm{ab}, \mathrm{A}}$ & $80,93^{\mathrm{ab}, \mathrm{A}}$ & $81,43^{\mathrm{ab}, \mathrm{A}}$ & $78,70^{\mathrm{b}, \mathrm{AB}}$ & $79,01^{\mathrm{ab}, \mathrm{A}}$ & $80,73^{\mathrm{ab}, A}$ \\
\hline
\end{tabular}

* Médias seguidas da mesma letra minúscula na horizontal (comparação entre épocas) e da mesma letra maiúscula na vertical (comparação entre amostras) não diferem significativamente a $5 \%$ de probabilidade.

Tabela 4. Parâmetro a (negativo - verde) referente à cor das conservas de palmito (médias de três repetições analíticas).

\begin{tabular}{|c|c|c|c|c|c|c|c|}
\hline \multirow[t]{2}{*}{ Variedade } & \multicolumn{7}{|c|}{ Cor externa } \\
\hline & $1^{\mathrm{a}}$ época & $2^{\mathrm{a}}$ época & $3^{\mathrm{a}}$ época & $4^{\mathrm{a}}$ época & $5^{\mathrm{a}}$ época & $6^{\mathrm{a}}$ época & $7^{\mathrm{a}}$ época \\
\hline Real & $-2,26^{\mathrm{a}, \mathrm{B}}$ & $-1,59^{\mathrm{abc}, \mathrm{A}}$ & $-1,90^{\mathrm{ab}, \mathrm{A}}$ & $-1,18^{\mathrm{bc}, \mathrm{B}}$ & $-1,19^{\mathrm{bc}, \mathrm{B}}$ & $-1,80^{\mathrm{ab}, \mathrm{B}}$ & $-0,91^{\mathrm{c}, \mathrm{B}}$ \\
\hline Açaí & $-1,42^{\mathrm{a}, \mathrm{C}}$ & $-0,48^{\mathrm{b}, \mathrm{B}}$ & $-0,81^{\mathrm{b}, \mathrm{B}}$ & $-1,00^{\mathrm{b}, \mathrm{B}}$ & $-0,96^{\mathrm{ab}, \mathrm{B}}$ & $-0,90^{\mathrm{ab}, \mathrm{C}}$ & $-0,76^{\mathrm{b}, \mathrm{B}}$ \\
\hline \multirow[t]{2}{*}{ Pupunha } & $-3,13^{\mathrm{a}, \mathrm{A}}$ & $-1,82^{\mathrm{c}, \mathrm{A}}$ & $-2,44^{\mathrm{b}, \mathrm{A}}$ & $-2,69^{\mathrm{ab}, \mathrm{A}}$ & $-3,05^{\mathrm{a}, \mathrm{A}}$ & $-3,25^{\mathrm{a}, \mathrm{A}}$ & $-2,97^{\mathrm{ab}, A}$ \\
\hline & \multicolumn{7}{|c|}{ Cor interna } \\
\hline Real & $-2,71^{\mathrm{a}, \mathrm{B}}$ & $-2,66^{\mathrm{a}, \mathrm{B}}$ & $-1,89^{\mathrm{ab}, \mathrm{B}}$ & $-1,96^{\mathrm{ab}, \mathrm{B}}$ & $-1,56^{\mathrm{ab}, \mathrm{B}}$ & $-2,21^{\mathrm{a}, \mathrm{B}}$ & $-0,93^{\mathrm{b}, \mathrm{C}}$ \\
\hline Açaí & $-3,32^{\mathrm{a}, \mathrm{B}}$ & $-2,53^{\mathrm{ab}, \mathrm{B}}$ & $-3,58^{\mathrm{a}, \mathrm{A}}$ & $-2,75^{\mathrm{ab}, \mathrm{B}}$ & $-2,41^{\mathrm{ab}, \mathrm{AB}}$ & $-2,13^{\mathrm{ab}, \mathrm{B}}$ & $-1,94^{\mathrm{b}, \mathrm{B}}$ \\
\hline Pupunha & $-4,35^{\mathrm{a}, \mathrm{A}}$ & $-3,88^{\mathrm{ab}, \mathrm{A}}$ & $-3,42^{\mathrm{b}, \mathrm{A}}$ & $-4,43^{\mathrm{a}, \mathrm{A}}$ & $-3,41^{\mathrm{b}, \mathrm{A}}$ & $-3,84^{\mathrm{ab}, \mathrm{A}}$ & $-4,61^{\mathrm{a}, \mathrm{A}}$ \\
\hline
\end{tabular}

*Médias seguidas da mesma letra minúscula na horizontal (comparação entre épocas) e da mesma letra maiúscula na vertical (comparação entre amostras) não diferem significativamente a $5 \%$ de probabilidade.

Tabela 5. Parâmetro b (positivo - cor amarela) referente à cor das conservas de palmito (médias de três repetições analíticas).

\begin{tabular}{|c|c|c|c|c|c|c|c|}
\hline \multirow[t]{2}{*}{ Variedade } & \multicolumn{7}{|c|}{ Cor externa } \\
\hline & $1^{\mathrm{a}}$ época & $2^{\mathrm{a}}$ época & $3^{\text {a época }}$ & $4^{\mathrm{a}}$ época & $5^{\mathrm{a}}$ época & $6^{\mathrm{a}}$ época & $7^{\mathrm{a}}$ época \\
\hline Real & $8,15^{\mathrm{bc}, \mathrm{B}}$ & $8,89^{\mathrm{bc}, \mathrm{B}}$ & $7,31^{\mathrm{c}, \mathrm{B}}$ & $8,45^{\mathrm{bc}, \mathrm{B}}$ & $8,84^{\mathrm{bc}, \mathrm{B}}$ & $9,88^{\mathrm{ab}, \mathrm{B}}$ & $10,89^{\mathrm{a}, \mathrm{B}}$ \\
\hline Açaí & $10,45^{\mathrm{b}, \mathrm{A}}$ & $12,52^{\mathrm{a}, \mathrm{A}}$ & $11,84^{\mathrm{ab}, \mathrm{A}}$ & $12,60^{\mathrm{a}, \mathrm{A}}$ & $13,11^{\mathrm{a}, \mathrm{A}}$ & $12,47^{\mathrm{a}, \mathrm{A}}$ & $13,06^{\mathrm{a}, \mathrm{A}}$ \\
\hline \multirow[t]{2}{*}{ Pupunha } & $10,06^{\mathrm{c}, \mathrm{A}}$ & $10,28^{\mathrm{bc}, \mathrm{B}}$ & $10,22^{\mathrm{bc}, \mathrm{A}}$ & $12,28^{\mathrm{abc}, \mathrm{A}}$ & $11,91^{\mathrm{ab} c, \mathrm{~A}}$ & $12,56^{\mathrm{ab}, \mathrm{A}}$ & $13,48^{\mathrm{a}, \mathrm{A}}$ \\
\hline & \multicolumn{7}{|c|}{ Cor interna } \\
\hline Real & $7,76^{\mathrm{d}, \mathrm{B}}$ & $10,10^{\mathrm{bcd}, \mathrm{B}}$ & $8,32^{\mathrm{cd}, \mathrm{B}}$ & $11,31^{\mathrm{abc}, \mathrm{B}}$ & $12,35^{\mathrm{ab}, \mathrm{B}}$ & $11,88^{\mathrm{ab}, \mathrm{B}}$ & $13,40^{\mathrm{a}, \mathrm{B}}$ \\
\hline Açaí & $13,63^{\mathrm{a}, \mathrm{A}}$ & $14,52^{\mathrm{a}, \mathrm{A}}$ & $17,84^{\mathrm{a}, \mathrm{A}}$ & $16,43^{\mathrm{a}, \mathrm{A}}$ & $17,29^{\mathrm{a}, \mathrm{A}}$ & $16,94^{\mathrm{a}, \mathrm{A}}$ & $16,06^{\mathrm{a}, \mathrm{Al}}$ \\
\hline Pupunha & $15,77^{\mathrm{b}, \mathrm{A}}$ & $16,97^{\mathrm{ab}, \mathrm{A}}$ & $15,67^{\mathrm{b}, \mathrm{A}}$ & $18,91^{\mathrm{a}, \mathrm{A}}$ & $17,26^{\mathrm{ab}, \mathrm{A}}$ & $17,89^{\mathrm{ab}, \mathrm{A}}$ & $19,12^{\mathrm{a}, \mathrm{A}}$ \\
\hline
\end{tabular}

*Médias seguidas da mesma letra minúscula na horizontal (comparação entre épocas) e da mesma letra maiúscula na vertical (comparação entre amostras) não diferem significativamente a $5 \%$ de probabilidade. 
o palmito da palmeira Real, em termos de coloração, assemelhase ao palmito da palmeira Açaí do gênero Euterpe.

\subsection{Avaliação de textura}

Os resultados das análises de textura nas diferentes épocas estão na Tabela 6. Os valores médios de textura dos toletes indicam diferenças quanto à maciez do produto. Neste caso, as medidas obtidas nos toletes apresentam valores muito superiores aos obtidos para as rodelas, tal fato ocorre devido à influência do sentido das fibras do tolete de palmito, ou seja, para as medidas tomadas nos toletes, o corte ocorre de forma perpendicular e no sentido contrário ao das fibras, e para as rodelas, perpendicularmente, mas no mesmo sentido das fibras.

Para todas as épocas de armazenamento, o palmito Pupunha apresentou maior maciez, seguido pelas palmeiras Real e Açaí. Estes resultados indicam que comparativamente o palmito Pupunha apresenta tecido mais macio e, conseqüentemente, necessita de um tempo menor de cozimento.

\subsection{Avaliação sensorial}

Os resultados da avaliação sensorial das conservas de palmito, nas diferentes épocas, estão apresentados nas Tabelas 7, 8, 9, 10 e 11.

Os resultados apresentados pela Tabela 7 indicam que quanto à avaliação entre as épocas de armazenamento, o palmito Pupunha avaliado na primeira época diferiu significativamente das amostras avaliadas nas demais épocas. De maneira geral, todas as amostras apresentaram médias superiores a 5, correspondendo ao termo "gostei" na escala utilizada. Estes valores indicam que do ponto de vista de cor subjetiva, palmitos da palmeira Real e Açaí escureceram durante os 12 meses de armazenamento. Estes resultados reforçam os resultados obtidos na avaliação objetiva de cor.

Os resultados de cor e aparência coincidem, sendo que as menores médias foram obtidas para o palmito Pupunha, seguido do Açaí e Real. Para os atributos de cor e aparência, o palmito da palmeira Real obteve as maiores médias $(6,17 ; 6,08 ; 4,42 ; 5,42)$, que corresponderam ao termo "gostei" da escala utilizada.

Para o atributo textura, muito importante para o produto em questão, as amostras avaliadas obtiveram médias correspondentes ao intervalo de termos "gostei" e "gostei muito" da escala utilizada, indicando muito boa aceitação do produto.
Tabela 7. Resultados da avaliação sensorial de cor.*

\begin{tabular}{cccc}
\hline Época & \multicolumn{3}{c}{ Variedades } \\
\cline { 2 - 4 } & Real & Açaí & Pupunha \\
\hline $1^{\mathrm{a}}$ & $6,42^{\mathrm{a}, \mathrm{A}}$ & $6,17^{\mathrm{a}, \mathrm{A}}$ & $4,42^{\mathrm{b}, \mathrm{A}}$ \\
$2^{\mathrm{a}}$ & $6,17^{\mathrm{a}, \mathrm{A}}$ & $5,58^{\mathrm{a}, \mathrm{A}}$ & $5,67^{\mathrm{a}, \mathrm{A}}$ \\
$3^{\mathrm{a}}$ & $6,42^{\mathrm{a}, \mathrm{A}}$ & $5,50^{\mathrm{a}, \mathrm{A}}$ & $4,83^{\mathrm{a}, \mathrm{A}}$ \\
$4^{\mathrm{a}}$ & $5,58^{\mathrm{a}, \mathrm{A}}$ & $5,17^{\mathrm{a}, \mathrm{A}}$ & $5,17^{\mathrm{a}, \mathrm{A}}$ \\
\hline
\end{tabular}

* Notas seguidas da mesma letra minúscula na horizontal (comparação entre amostras) e da mesma letra maiúscula na vertical (comparação entre épocas) não diferem significativamente a $5 \%$ de erro.

Tabela 8. Resultados da avaliação sensorial de aparência.*

\begin{tabular}{cccc}
\hline Época & \multicolumn{3}{c}{ Variedades } \\
\cline { 2 - 4 } & Real & Açaí & Pupunha \\
\hline $1^{\mathrm{a}}$ & $6,17^{\mathrm{a}, \mathrm{A}}$ & $5,92^{\mathrm{a}, \mathrm{A}}$ & $4,67^{\mathrm{b}, \mathrm{A}}$ \\
$2^{\mathrm{a}}$ & $6,08^{\mathrm{a}, \mathrm{A}}$ & $5,75^{\mathrm{a}, \mathrm{A}}$ & $5,58^{\mathrm{a}, \mathrm{A}}$ \\
$3^{\mathrm{a}}$ & $4,42^{\mathrm{a}, \mathrm{B}}$ & $5,50^{\mathrm{a}, \mathrm{A}}$ & $4,83^{\mathrm{a}, \mathrm{A}}$ \\
$4^{\mathrm{a}}$ & $5,42^{\mathrm{a}, \mathrm{AB}}$ & $5,25^{\mathrm{a}, \mathrm{A}}$ & $5,67^{\mathrm{a}, \mathrm{A}}$ \\
\hline
\end{tabular}

*Notas seguidas da mesma letra minúscula na horizontal (comparação entre amostras) e da mesma letra maiúscula na vertical (comparação entre épocas) não diferem significativamente a $5 \%$ de erro.

Tabela 9. Resultados da avaliação sensorial de textura.*

\begin{tabular}{cccc}
\hline Época & \multicolumn{3}{c}{ Variedade } \\
\cline { 2 - 4 } & Real & Açaí & Pupunha \\
\hline $1^{\mathrm{a}}$ & $5,75^{\mathrm{a}, \mathrm{A}}$ & $6,00^{\mathrm{a}, \mathrm{A}}$ & $5,42^{\mathrm{a}, \mathrm{A}}$ \\
$2^{\mathrm{a}}$ & $6,17^{\mathrm{a}, \mathrm{A}}$ & $5,50^{\mathrm{a}, \mathrm{A}}$ & $5,67^{\mathrm{a}, \mathrm{A}}$ \\
$3^{\mathrm{a}}$ & $6,00^{\mathrm{a}, \mathrm{A}}$ & $6,00^{\mathrm{a}, \mathrm{A}}$ & $5,33^{\mathrm{ab}, \mathrm{A}}$ \\
$4^{\mathrm{a}}$ & $5,67^{\mathrm{a}, \mathrm{A}}$ & $5,33^{\mathrm{a}, \mathrm{A}}$ & $5,17^{\mathrm{a}, \mathrm{A}}$ \\
\hline
\end{tabular}

${ }^{*}$ Notas seguidas da mesma letra minúscula na horizontal (comparação entre amostras) e da mesma letra maiúscula na vertical (comparação entre épocas) não diferem significativamente a $5 \%$ de erro.

Tabela 10. Resultados da avaliação sensorial de sabor.*

\begin{tabular}{cccc}
\hline Época & \multicolumn{3}{c}{ Variedade } \\
\cline { 2 - 4 } & Real & Açaí & Pupunha \\
\hline $1^{\mathrm{a}}$ & $5,92^{\mathrm{ba}, \mathrm{A}}$ & $6,00^{\mathrm{a}, \mathrm{A}}$ & $4,83^{\mathrm{b}, \mathrm{A}}$ \\
$2^{\mathrm{a}}$ & $6,08^{\mathrm{a}, \mathrm{A}}$ & $5,92^{\mathrm{a}, \mathrm{A}}$ & $5,25^{\mathrm{a}, \mathrm{A}}$ \\
$3^{\mathrm{a}}$ & $6,00^{\mathrm{a}, \mathrm{A}}$ & $6,00^{\mathrm{a}, \mathrm{A}}$ & $4,92^{\mathrm{a}, \mathrm{A}}$ \\
$4^{\mathrm{a}}$ & $5,25^{\mathrm{a}, \mathrm{A}}$ & $5,25^{\mathrm{a}, \mathrm{A}}$ & $4,42^{\mathrm{a}, \mathrm{A}}$ \\
\hline
\end{tabular}

${ }^{*}$ Notas seguidas da mesma letra minúscula na horizontal (comparação entre amostras) e da mesma letra maiúscula na vertical (comparação entre épocas) não diferem significativamente a $5 \%$ de erro.

Tabela 6. Textura do palmito em conserva em gf (médias de seis repetições analíticas).*

\begin{tabular}{|c|c|c|c|c|c|c|c|}
\hline \multirow[t]{2}{*}{ Variedade } & \multicolumn{7}{|c|}{ Textura externa } \\
\hline & $1^{\mathrm{a}}$ época & $2^{\mathrm{a}}$ época & $3^{\mathrm{a}}$ época & $4^{\mathrm{a}}$ época & $5^{\mathrm{a}}$ época & 6a época & 7a época \\
\hline Real & $5,06^{\mathrm{b}, \mathrm{AB}}$ & $6,57^{\mathrm{ab}, \mathrm{AB}}$ & $5,04^{\mathrm{b}, \mathrm{AB}}$ & $10,55^{\mathrm{a}, \mathrm{A}}$ & $8,27^{\mathrm{ab}, \mathrm{A}}$ & $7,25^{\mathrm{ab}, \mathrm{A}}$ & $5,79^{\mathrm{ab}, \mathrm{A}}$ \\
\hline Açaí & $6,68^{\mathrm{b}, \mathrm{A}}$ & $11,75^{\mathrm{a}, \mathrm{A}}$ & $8,18^{\mathrm{ab}, \mathrm{A}}$ & $9,40^{\mathrm{ab}, \mathrm{B}}$ & $9,71^{\mathrm{ab}, \mathrm{A}}$ & $9,51^{\mathrm{ab}, \mathrm{A}}$ & $8,44^{\mathrm{ab}, \mathrm{A}}$ \\
\hline \multirow[t]{2}{*}{ Pupunha } & $3,56^{\mathrm{a}, \mathrm{B}}$ & $3,25^{\mathrm{a}, \mathrm{B}}$ & $3,97^{\mathrm{a}, \mathrm{B}}$ & $4,71^{\mathrm{a}, \mathrm{B}}$ & $5,07^{\mathrm{a}, \mathrm{B}}$ & $7,35^{\mathrm{a}, \mathrm{A}}$ & $5,52^{\mathrm{a}, \mathrm{A}}$ \\
\hline & \multicolumn{7}{|c|}{ Textura interna } \\
\hline Real & $0,42^{\mathrm{b}, \mathrm{AB}}$ & $1,29^{\mathrm{a}, \mathrm{A}}$ & $0,69^{\mathrm{b}, \mathrm{B}}$ & $1,03^{\mathrm{ab}, \mathrm{A}}$ & $1,11^{\mathrm{ab}, \mathrm{A}}$ & $1,30^{\mathrm{a}, \mathrm{A}}$ & $13,40^{\mathrm{a}, \mathrm{B}}$ \\
\hline Açaí & $1,10^{\mathrm{a}, \mathrm{A}}$ & $1,13^{\mathrm{a}, \mathrm{A}}$ & $1,21^{\mathrm{a}, \mathrm{A}}$ & $1,46^{\mathrm{a}, \mathrm{A}}$ & $1,32^{\mathrm{a}, \mathrm{A}}$ & $1,78^{\mathrm{a}, \mathrm{A}}$ & $16,06^{\mathrm{a}, \mathrm{AB}}$ \\
\hline Pupunha & $0,36^{\mathrm{b}, \mathrm{B}}$ & $0,56^{\mathrm{ab}, \mathrm{B}}$ & $0,81^{\mathrm{ab}, \mathrm{AB}}$ & $0,65^{\mathrm{ab}, \mathrm{B}}$ & $1,11^{\mathrm{ab}, \mathrm{A}}$ & $1,46^{\mathrm{a}, \mathrm{A}}$ & $19,12^{\mathrm{a}, \mathrm{A}}$ \\
\hline
\end{tabular}

${ }^{\star}$ Médias seguidas da mesma letra minúscula na horizontal (comparação entre épocas) e da mesma letra maiúscula na vertical (comparação entre amostras) não diferem significativamente a $5 \%$ de probabilidade. 
Tabela 11. Resultados da avaliação sensorial de preferência geral. ${ }^{*}$

\begin{tabular}{cccc}
\hline Época & \multicolumn{3}{c}{ Variedade } \\
\cline { 2 - 4 } & Real & Açaí & Pupunha \\
\hline $1^{\mathrm{a}}$ & $6,00^{\mathrm{a}, \mathrm{A}}$ & $5,92^{\mathrm{a}, \mathrm{A}}$ & $4,83^{\mathrm{b}, \mathrm{A}}$ \\
$2^{\mathrm{a}}$ & $5,75^{\mathrm{a}, \mathrm{A}}$ & $5.75^{\mathrm{a}, \mathrm{A}}$ & $5.41^{\mathrm{a}, \mathrm{A}}$ \\
$3^{\mathrm{a}}$ & $5,52^{\mathrm{a}, \mathrm{A}}$ & $5,75^{\mathrm{a}, \mathrm{A}}$ & $5,17^{\mathrm{a}, \mathrm{A}}$ \\
$4^{\mathrm{a}}$ & $5,33^{\mathrm{a}, \mathrm{A}}$ & $5,25^{\mathrm{a}, \mathrm{A}}$ & $4,67^{\mathrm{a}, \mathrm{A}}$ \\
\hline
\end{tabular}

${ }^{*}$ Notas seguidas da mesma letra minúscula na horizontal (comparação entre amostras) e da mesma letra maiúscula na vertical (comparação entre épocas) não diferem significativamente a $5 \%$ de erro.

Estes resultados coincidem com a avaliação de sabor apresentada na Tabela 10.

Os resultados do atributo "preferência geral" apresentados na Tabela 11 mostram que os produtos apresentaram médias correspondentes ao intervalo de termos "gostei" e "gostei muito". O palmito Pupunha apresentou diferença significativa nas comparações entre amostras e épocas de avaliação.

Do ponto de vista da avaliação sensorial, o palmito da palmeira Real não apresentou diferença significativa dos palmitos das variedades Pupunha e Açaí e se manteve sensorialmente estável durante o período de armazenamento. Estes resultados em conjunto com os resultados físico-químicos, de cor e textura objetiva indicam que uma vida de prateleira de 12 meses para o palmito da palmeira Real em conserva acidificada e pasteurizada é aceitável.

\section{Conclusões}

A partir dos resultados encontrados neste estudo, pode-se concluir que:

- Do ponto de vista da estabilidade ao armazenamento, o palmito da palmeira Real em conserva apresentou-se estável durante o período de doze meses de armazenamento, conforme demonstraram os resultados das análises físico-químicas e microbiológicas;

- O palmito da palmeira Real processado na forma de conserva acidificada e pasteurizada apresenta características sensoriais semelhantes às das variedades Açaí e Pupunha tradicionalmente utilizadas para este fim; $\mathrm{e}$
- Desta forma, pode-se afirmar que a palmeira Real Australiana apresenta boa qualidade em termos de industrialização na forma de palmito em conserva acidificada e pasteurizada.

\section{Referências bibliográficas}

AOAC - Association of Official Analytical Chemists. Official methods of analysis. $16^{\text {th }} \mathrm{ed}$. Washington: AOAC, 1997.

. Official methods of analysis. Edited by Patricia Cunniff. $17^{\text {th }}$ ed. Washington: AOAC, 2000.

BOVI, M. L. A. Cultivo da palmeira Real Australiana visando à produção de palmito. Campinas: IAC, 1998. (Boletim técnico, n. 172).

BOVI, M. L. A. Palmito Pupunha: Informações básicas para o cultivo. In: PASCHOALINO, J. E. et al. Industrialização do palmito Pupunha. Campinas: ITAL, 1997. Cap.1, p.1-14. (Manual Técnico, n. 15)

DIEMAIR, W. Laboratorium sbuch fur lebensmittel - chemiker. Drisden: Verlag Von Theodor Steinkopff, 1963.

DOWNES, F. P.; ITO, K. (ed.). Compendium of methods for the microbiological examination of foods. $4^{\text {th }} \mathrm{ed}$. Washington: American Public Health Association, 2001.

FARIA, E. V.; YOTSUYANAGI, K. Técnicas de análise sensorial. Campinas: ITAL/LAFISE, 2002.

FERREIRA, V. L. P. et al. Comparação entre os palmitos de Guilielma gasipaes Bailey (Pupunha) e Euterpe Edulis Mart. (Juçara). I Avaliações físicas, organolépticas e bioquímicas. Coletânea do ITAL, n. 12, p. 255-272, 1981/1982.

IAL - Instituto Adolfo Lutz. Normas analíticas do Instituto Adolfo Lutz: métodos químicos e físicos para análise de alimentos. 4 ed. v. 1. São Paulo: Instituto Adolfo Lutz, 2005.

ISO - International Organization for Standardization. Sensory analysis: methodology - triangular test. Swtzerland: ISO 4120, 1983.

MORI, E. E. M. et al. Seleção de parâmetros que definem a qualidade sensorial de palmito em conserva. Coletânea do ITAL, v. 19, n. 1, p. 77-100, jan/jun, 1989.

TAUSZIG, L. Controle e medição de cor a aparência na indústria de alimentos. In: MOURA, S. C. S. R.; VISSOTO, F. Z. Seminário sobre propriedades termofísicas aplicadas na garantia da qualidade de alimentos. Campinas: ITAL, 2000.

ZAPATA, J. M.; QUAST, D. G. Curvas de titulação de palmito doce. Coletânea do ITAL, v. 6, n. 1, p. 167-168, 1975. 